\title{
Neuromuscular blockers and ARDS: Thou shalt not breathe, move, or die!
}

\author{
Balaji Yegneswaran' and Raghavan Murugan ${ }^{1,2}$ \\ University of Pittsburgh Department of Critical Care Medicine: Evidence-Based Medicine Journal Club, edited by Sachin Yende
}

\section{Expanded abstract Citation \\ Papazian L, Forel JM, Gacouin A, Penot-Ragon C, Perrin G, Loundou A, Jaber S, Arnal JM, Perez D, Seghboyan JM, Constantin JM, Courant P, Lefrant JY, Guérin C, Prat G, Morange S, Roch A; ACURASYS Study Investigators: Neuromuscular blockers in early acute respiratory distress syndrome. $N$ Engl J Med 2010, 363:1107-16. PMID:20843245.}

\section{Background}

In patients undergoing mechanical ventilation for Acute Respiratory Distress Syndrome (ARDS), neuromuscular blocking agents (NMBAs) may improve oxygenation and decrease ventilator-induced lung injury but may also cause muscle weakness.

\section{Methods}

Objective: To identify if 48 hour therapy with the NMBA cisatracurium early in the course of ARDS reduces adjusted 90-day in-hospital mortality rate.

Design: Multicenter, double blind, randomized clinical trial.

Setting: Twenty multidisciplinary intensive care units in France

Subjects: Patients presenting with onset of severe ARDS within the previous 48 hours. Severe ARDS was defined as a ratio of the partial pressure of arterial oxygen to the fraction of inspired oxygen of less than 150, with a positive end-expiratory pressure of $5 \mathrm{~cm}$ or more of water and a tidal volume of 6 to $8 \mathrm{ml}$ per kilogram of predicted body weight.

Intervention: After enrollment, 340 patients were randomized to receive either cisatracurium besylate $(n=178)$ or placebo $(n=162)$. All patients were sedated to a Ramsay

*Correspondence: muruganr@ccm.upmc.edu

'Department of Critical Care Medicine, University of Pittsburgh School of Medicine, Pittsburgh, PA

Full list of author information is available at the end of the article sedation score of 6 using sulfentanil and midazolam prior to intervention. A dose of $15 \mathrm{mg}$ cisatracurium besylate or placebo was then administered, followed by a continuous infusion of $37.5 \mathrm{mg} /$ hour for 48 hours. Patients were not monitored for depth of paralysis.

Outcomes: The primary outcome was the proportion of patients who died either before hospital discharge or within 90 days after study enrollment (i.e., the 90-day inhospital mortality rate), adjusted for predefined covariates and baseline differences between groups with the use of a Cox model.

\section{Results}

The crude 90-day mortality was $31.6 \%$ (95\% confidence interval [CI], 25.2 to 38.8) in the cisatracurium group and $40.7 \%$ (95\% CI, 33.5 to 48.4 ) in the placebo group $(P=0.08)$. The hazard ratio for death at 90 days in the cisatracurium group, as compared with the placebo group, was 0.68 ( $95 \% \mathrm{CI}, 0.48$ to $0.98 ; P=0.04$ ), after adjustment for the baseline $\mathrm{PaO}_{2}: \mathrm{FIO}_{2}$ ratio, plateau pressure and the Simplified Acute Physiology II score. Mortality at 28 days was $23.7 \%$ (95\% CI, 18.1 to 30.5 ) with cisatracurium and $33.3 \%$ (95\% CI, 26.5 to 40.9 ) with placebo $(P=0.05)$. The rate of ICU-acquired paresis did not differ significantly between the two groups.

\section{Conclusions}

In patients with severe ARDS, early administration of a neuromuscular blocking agent improved the adjusted 90-day survival and increased the time off the ventilator without increasing muscle weakness.

\section{Trial Registration}

NCT00299650

\section{Commentary}

The incidence of ARDS varies between 13.5 to 58.7 cases per 100,000 person years [1] , and affects $10 \%$ to $15 \%$ of all patients admitted to the intensive care unit $[2,3]$. Despite various treatment options, such as high positive endexpiratory pressure, corticosteroids, recruitment maneuvers, conservative fluid management, and rescue 


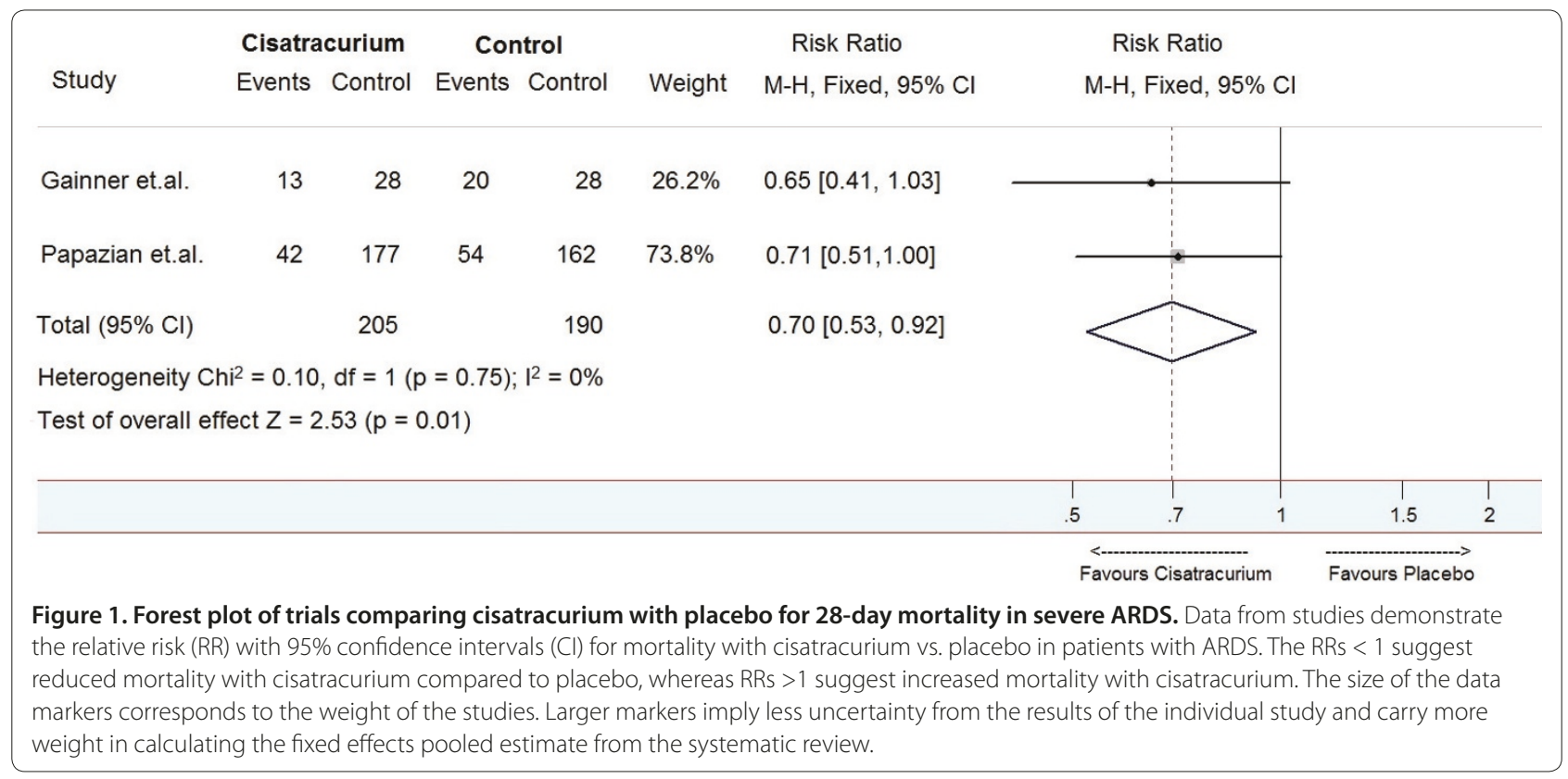

maneuvers, including prone ventilation, nitric oxide, and high frequency oscillation ventilation, severe ARDS has a mortality ranging from $40 \%$ to $60 \%$ [2,3]. Only low tidal volume ventilation and extracorporeal membrane oxygenation have been shown to decrease mortality in patients with ARDS $[4,5]$.

Neuromuscular blocking agents are currently used as a salvage maneuver in patients with severe ARDS in whom oxygenation is a challenge. Use of NMBAs has been hypothesized to achieve better synchronization with the ventilator resulting in decreased ventilator-induced lung injury, and improve oxygenation due to lower oxygen consumption [6]. NMBAs are also thought to have an anti-inflammatory effect, including attenuation of interleukin-6 and 8 expression [6]. However, NMBAs preclude the ability to monitor neurological signs clinically and prolonged use has been associated with increased risk of critical illness polyneuropathy [7], and posttraumatic stress disorder symptoms when compared to patients managed with sedatives alone [8]. Therefore, the current standard of practice in the intensive care unit is to minimize the use of sedation, wake patients early, and avoid use of paralytic medications. Indeed, the Society of Critical Care Medicine guidelines recommend that NMBAs should only be used to manage ventilation and decrease oxygen consumption when all other means have been tried without success (Grade $\mathrm{C}$ recommendation) [9].

Papazian and colleagues challenge this existing paradigm and ask a very intriguing question whether short term use of NMBAs is likely to improve outcomes from ARDS. The authors in an earlier smaller study found that early and short course of NMBA use were associated with a trend toward lower 28-day mortality [10]. In the current study the authors found that in patients with severe ARDS, early administration of cisatracurium improved the adjusted 90-day survival and increased the time off the ventilator without increasing muscle weakness. Strengths of the trial include a well-defined study protocol, multicenter design and early assignment to treatment groups, intention-to-treat analysis, and complete follow-up.

However, there are several important limitations that deserve consideration. First, although the healthcare providers were blinded to the best possible extent, complete blinding would not be possible as physicians can easily identify a paralyzed patient (by a thorough clinical exam). This could have introduced a systematic bias in the intervention arm that could have influenced the outcomes. Second, it is important to note that the authors used hospital mortality censored at 90 days as a primary outcome measure. However, the abstract as well as the remainder of the manuscript alludes to "90-day" mortality rate as a primary outcome. The distinction is important because "90-day mortality" (i.e., assessing whether a patient is alive or dead at 90 days following randomization irrespective of their location) is a more robust outcome measure than hospital mortality censored at 90 days (i.e., assessing mortality only during hospital stay within the first 90 days of randomization) as the latter does not account for patients who might have died following hospital discharge within the first 90 days. Using in-hospital mortality rates can also make it difficult to interpret outcome differences across institutions if the 
discharge policies of these institutions vary. For example, transfer of ICU patients to long term acute care facilities, or earlier hospital discharges, may cause a decrease in the hospital mortality rate.

Third, although the authors noted a low risk of barotrauma and organ failure with NMBA use, data related to mechanistic pathways (e.g., decrease in biomarkers) that could have mediated beneficial outcome is lacking. Fourth, as stated by the authors the study was underpowered to detect differences in crude mortality rates and statistical significance was reached only after adjustment for baseline covariates. Finally, the authors used the Medical Research Council scale to evaluate muscle weakness at 28 days, which may be too brief to recognize muscle weakness especially among patients who require prolonged mechanical ventilation. Assessing long term neuromuscular weakness related to NMBA use [11], is likely to provide more robust safety data related to NMBA use.

This trial challenges current prevailing practice paradigms of whether a minimalistic approach in the treatment of ARDS, such as use of lighter sedation and no paralytic agents, improves outcomes. Nevertheless, this is the second study showing that short course NMBA may improve outcome among patients with severe ARDS $[10,12]$. We conducted an analysis from the two studies by pooling crude estimates of treatment effects of NMBA on 28-day mortality [10,12] (Figure 1). We found that the use of NMBA cisatracurium lowered mortality rate (Relative Risk $=0.7,95 \%$ CI, 0.53-0.92, $P=0.01$ ) compared to placebo. However, the caveat should be noted that the 28-day mortality is a suboptimal outcome measure for assessing interventions in ARDS [13].

\section{Recommendation}

Papazian and colleagues should be commended for showing that early and short term administration of NMBAs is safe, may improve mortality, decrease duration of mechanical ventilation, and complications related to barotrauma. The absence of significant short term adverse effects and the potential to improve mortality, although needs to be replicated in future studies, suggest that early and short course of NMBAs may be beneficial in severe ARDS.

Competing interests

The authors declare that they have no competing interests.

\section{Author details}

'Department of Critical Care Medicine, University of Pittsburgh School of Medicine, Pittsburgh, PA. ${ }^{2}$ The Clinical Research, Investigation, and Systems Modeling of Acute IIIness (CRISMA) Center, University of Pittsburgh School of Medicine, Pittsburgh, PA

Published: 30 September 2011

References

1. Frutos-Vivar F, Nin N, Esteban A: Epidemiology of acute lung injury and acute respiratory distress syndrome. Curr Opin Crit Care 2004, 10:1-6.

2. Luhr OR, Antonsen $K$, Karlsson M et al:: Incidence and mortality after acute respiratory failure and acute respiratory distress syndrome in Sweden, Denmark, and Iceland. The ARF Study Group. Am J Respir Crit Care Med 1999, 159:1849-1861.

3. Rubenfeld GD, Caldwell E, Peabody E et al:. Incidence and outcomes of acute lung injury. N Engl J Med 2005, 353:1685-1693.

4. Esan A, Hess DR, Raoof S, George L, Sessler CN: Severe hypoxemic respiratory failure: part 1 - ventilatory strategies. Chest 2010, 137:1203-1216.

5. Raoof S, Goulet K, Esan A, Hess DR, Sessler CN: Severe hypoxemic respiratory failure: part 2 - nonventilatory strategies. Chest 2010, 137:1437-1448.

6. Marini JJ: Early phase of lung-protective ventilation: a place for paralytics? Crit Care Med 2006, 34:2851-2853.

7. Garnacho-Montero J, Madrazo-Osuna J, Garcia-Garmendia JL et al.: Critical illness polyneuropathy: risk factors and clinical consequences. A cohort study in septic patients. Intensive Care Med 2001, 27:1288-1296.

8. Nelson BJ, Weinert CR, Bury CL, Marinelli WA, Gross CR: Intensive care unit drug use and subsequent quality of life in acute lung injury patients. Crit Care Med 2000, 28:3626-3630.

9. Murray MJ, Cowen J, DeBlock H et al:: Clinical practice guidelines for sustained neuromuscular blockade in the adult critically ill patient. Crit Care Med 2002, 30:142-156.

10. Gainnier M, Roch A, Forel JM et al.: Effect of neuromuscular blocking agents on gas exchange in patients presenting with acute respiratory distress syndrome. Crit Care Med 2004, 32:113-119.

11. De JB, Sharshar T, Lefaucheur JP et al:: Paresis acquired in the intensive care unit: a prospective multicenter study. JAMA 2002, 288:2859-2867.

12. Papazian L, Forel JM, Gacouin A et al. Neuromuscular blockers in early acute respiratory distress syndrome. N Engl J Med 2010, 363:1107-1116.

13. Hebert PC, Cook DJ, Wells G, Marshall J: The design of randomized clinical trials in critically ill patients. Chest 2002, 121:1290-1300.

doi:10.1186/cc10470

Cite this article as: Yegneswaran B, Murugan R: Neuromuscular blockers and ARDS: Thou shalt not breathe, move, or die! Critical Care 2011, 15:311. 